\title{
Prevalence Trend of Diabetic Retinopathy in India
}

\section{OPEN ACCESS}

Manuscript ID:

ECO-2021-09034052

Volume: 9

Issue: 3

Month: June

Year: 2021

P-ISSN: 2319-961X

E-ISSN: 2582-0192

Received: 17.03.2021

Accepted: 29.04.2021

Published: 01.06.2021

Citation:

Chitra, Muthu, and

Aruthdha Shree

Neevanandam. "Prevalence

Trend of Diabetic

Retinopathy in India."

Shanlax International

Journal of Economics, vol. 9, no. 3, 2021, pp. 49-56.

DOI:

https://doi.org/10.34293/ economics.v9i3.4052

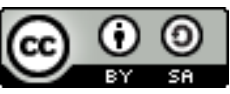

This work is licensed under a Creative Commons Attribution-ShareAlike 4.0 International License

\section{Chitra}

Assistant Professor, Department of Econometrics, Madurai Kamaraj University, Madurai, India https://orcid.org/0000-0002-6427-0988

\section{Aruthdha Shree Neevanandam}

Nantong University, Nantong, Jiangsu, China

\section{Abstract}

Background: Diabetic Retinopathy plays a vital role in the impact of long-term Diabetic patients in global humankind. The health of diabetic people determined by many factors, specifically the number of years of diabetic suffering. There is a positive relationship between the risk of diabetic retinopathy and the number of years of diabetic sufferings, which was identified by many researchers globally. In addition, patients who suffer from type 2 diabetes mellitus are suffering from diabetic retinopathy. However, there are few comprehensive reviews and studies focusing on its prevalence and the factors of prevalence. It is worthwile to pay attention to diabetic retinopathy in Global, India and its region, given that the trend and structure of diabetic retinopathy from reviews. Method: Prevalence provides a cross-sectional snapshot of morbidity at that point or period. The study is a concurrent review of diabetic retinopathy in India and its region. It presents the findings from some national or regional camp data, interviews with key informants, reviews of relevant published papers and policy contents. Results and Conclusion: Approximately one in five people living with diabetes in India has some degree of DR (13 million in India) and one in ten (6.5 million) has the vision-threatening form of DR. Tamil Nadu is the topmost in the prevalence rate of Diabetic Retinopathy among the states of India with above 10.5 percentage based on the report of Amaltas 2019. Hence, a mass survey for diabetic retinopathy screening needed to be conducted in all districts to know the exact status and plan in the National Programme for Control of Blindness and Visual Impairment $(N P C B \& V I)$.

Keywords: Diabetic retinopathy, Prevalence, Diabetes mellitus

\section{Introduction}

Diabetes mellitus is a disorder of carbohydrate metabolism characterized by impaired ability of the body to produce or respond to insulin and thereby maintain proper levels of sugar (glucose) in the blood. There are three main types of diabetes as type 1 diabetes (T1D), type 2 diabetes mellitus (T2D), and gestational diabetes mellitus (GDM). According to Pouya Saeedi et al., (2019), the general global diabetes prevalence in 2019 is estimated to be $9.3 \%$ (463 million people), rising to $10.2 \%$ (578 million) by 2030 and $10.9 \%$ (700 million) by 2045 . The prevalence is higher in urban $(10.8 \%)$ than rural $(7.2 \%)$ areas and in high-income (10.4\%) than low-income countries (4.0\%).

Further, International Diabetes Federation, Diabetes Atlas, defines diabetes prevalence. According to the federation definition diabetes prevalence refers to the percentage of people ages 20-79 who have type 1 or type 2 Diabetes. Based on this definition, 195 countries were ranked by the International Diabetes federation in 2019 for the prevalence rate of diabetes. In that ranking, India was ranked 46th place with 10.40, Malaysia was 16.7 in 12th place, China was 9.2 in 60th place, Thailand was 7.0 in 88th place, and Indonesia was 6.3 in 108th and Pakistan 19.9 in 6th place. So, Among the Asian countries, Pakistan is leading in the prevalence rate of diabetes, followed by Malaysia, India, China, Thailand \& Indonesia (International Diabetes Federation, Diabetes Atlas, 2019). 
Specifically, Type II Diabetes Mellitus (DM) is a major non-communicable disease which is alarmingly increasing in the past two decades. It is estimated that there will be a 195 percent increase in persons with diabetes by 2024 and hence is a major public health concern both in developed and developing countries. The estimated increase of DM is approximately from 30 million in 2000 to 80 million in 2030 in developing countries (Wild S, et al., 2004)

Diabetes of all types can lead to complications in many parts of the body and increase the overall risk of dying prematurely. Possible complications include heart attack, stroke, kidney failure, leg amputation, vision loss and nerve damage. In pregnancy, poorly controlled diabetes increases the risk of fetal death and other complications (Global report on diabetes: WHO 2016). Studies have shown that as the duration of diabetes increases, so does the chance of developing Diabetic Retinopathy. Diabetes and diabetic retinopathy have been emerging as a significant non-communicable disease leading to ocular morbidity. It is estimated that diabetic retinopathy was responsible for $1.06 \%$ of blindness and $1.16 \%$ of visual impairment globally in 2015 . There are no recent studies on the prevalence of diabetic retinopathy in different parts of India. This makes it difficult to identify where diabetic retinopathy screening and treatment programs are most needed, the government said. (Neetu Chandra Sharma live mint 10 Oct 2019). Hence, the researcher attempted to examine and present the trending prevalence of Diabetic retinopathy in India from different authors' research finding.

\section{Findings and Discussions}

Diabetic retinopathy (DR) is one of the commoner microvascular complications of diabetes, which occurs in both type 1 and type $2 \mathrm{DM}$. The risk increases with increasing duration of diabetes and poorly controlled hyperglycemia and hypertension (Ramakrishna et al., 2020). Diabetic retinopathy (DR) can be defined as damage to the microvascular system in the retina due to prolonged hyperglycemia. The pooled prevalence of Diabetic Retinopathy (DR), Proliferative Diabetic Retinopathy (PDR) and Non-Proliferative Diabetic Retinopathy (NPDR) estimated by review process in Asian type 2 diabetes mellitus (T2DM) patients. The systematic review and a meta-analysis study revealed that the prevalence of DR in T2DM patients from Singaporean, Indian, South Korean, Malaysian, Asian, and Chinese was $33 \%, 42 \%, 16 \%, 35 \%, 21 \%$ and $25 \%$, respectively. In T2DM patients with NPDR from Indian, South Korean, Malaysian, Asian, Chinese, higher prevalence was found than that in PDR patients ( $45 \%$ vs $17 \%, 13 \%$ vs $3 \%, 30 \%$ vs $5 \%, 23 \%$ vs $2 \%$ and $22 \%$ vs $3 \%$ ), as well as in DR patients ( $74 \%$ vs $26 \%, 81 \%$ vs $19 \%, 86 \%$ vs $14 \%, 92 \%$ vs $8 \%$ and $85 \%$ vs $15 \%$ ). The prevalence of PDR in T2DM from India was higher than patients from other locations of Asia (Yang QH, et al., 2019). India is home to over 74 million diabetics, and the number is estimated to exceed 123 million by 2040 (IDF 2015). The largest increase in the disease burden (among all non-communicable diseases) between 1996 and 2016 was noted for DM at $80 \%$ (Health report of India, 2017).

There is limited information on the prevalence of Diabetic Retinopathy (DR) among diabetic subjects and its associated factors in a rural setting in developing countries, including India (Balasubramaiyan, et al., 2017). India has a high burden of diabetic retinopathy ranging from $12.2 \%$ to $20.4 \%$ among patients with type 2 diabetes mellitus (T2DM). Diabetes and its complications bring about the substantial economic loss to people with diabetes and their families and health systems and national economies through direct medical costs and loss of work and wages. While the major cost drivers are hospital and outpatient care, a contributing factor is a rise in cost for analog insulins (Lancet, 2016). Particularly, the diabetic retinopathy opportunity cost is high in its affected one and their families.

A systematic literature search was carried out in Ovid Medline and EMBASE databases using Mesh and key search terms. Studies that reported the proportion of people with diabetes with DR in a representative community population were included. Two independent reviewers reviewed all the retrieved publications. Data were extracted using a predefined form. Review Manager Software was used to perform meta-analysis to provide a pooled estimate. Studies included were assessed for 
methodological quality using selected items from the STROBE checklist. Seven studies (1999-2014; $\mathrm{n}=8315$ persons with diabetes) were included in the review. In the meta-analysis, $14.9 \%$ (95\% confidence interval [CI] 10.7-19.0\%) of known diabetics aged $\geq 30$ years and $18.1 \%$ (95\% CI 14.8-21.4) among those aged $\geq 50$ years had DR. Heterogeneity around this estimate ranged from $\mathrm{I} 2=79-87 \%$. No linear trend was observed between age and the proportion with DR (Jotheeswaran et al. 2016)

A survey was conducted by Rajendra Prasad Center for Ophthalmic Sciences and AIIMS, New Delhi, in 2019 is the first survey to include Diabetic Retinopathy (DR) along with diabetes. DR was prevalent in the diabetic population aged up to 50 years. Around $16.9 \%$ of the population suffered from DR. Among males DR was prevalent among $17 \%$ of $16.7 \%$ of females DR among different age groups were observed as follows 60-69 years - 18.6\% 70-79 years $-18.3 \%$ Above 80 years - $18.4 \%$ Prevalence of blindness among diabetic patients was $2.1 \%$ and visual impairment was 13.7\% (Gadkarai et al., 2014).

In a cross-sectional hospital-based study, 500 patients with established diabetes who attended eye OPD at Govt Medical College Srinagar were evaluated for the presence or absence of retinopathy. Out of 500 patients, 135 patients had DR showing a prevalence of DR as $27 \%$. $62.2 \%$ of patients with DR were $>60$ years of age and 49 patients $(36.2 \%)$ were between 40-68 years of age. 33 (24.5\%) were males and $102(75.5 \%)$ were females. 30 patients $(12.8 \%)$ with diabetes of $=15$ year. Mild DR was present in 67 (37.4\%) patients, moderate to severe DR in 46 (9.2\%) patients, proliferative DR in $5(1 \%)$ patients and diabetic maculopathy in $17(3.4 \%)$ patients. Patients managed with insulin, either alone or with oral hypoglycemic drugs, had more prevalence of DR. (Tariq Qureshi, et al., 2013). A nationalwide cross-sectional study of diabetic patients was conducted as an initiative of the All India Ophthalmological Society from 14th November to 21 st November 2014. A total of 6218 diabetic patients across 194 centers were screened and finally, 5130 data entry forms were considered for further evaluation about retinopathy. The obtained results of a study about Diabetic Retinopathy (DR) prevalence in the entire data set was $21.7 \%$.
Prevalence was more in males $(\mathrm{P}=0.007)$, diabetics more than 5 years $(\mathrm{P}=0.001)$, those above 40 years $(\mathrm{P}=0.01)$, insulin users $(\mathrm{P}=0.001)$, and history of vascular accidents $(\mathrm{P}=0.0014)$. Significantly $22.18 \%$ of patients detected with DR had a vision of $6 / 18$ or better in the worse eye (Gadkari et al. 2014).

Diabetic Retinopathy (DR) is a high risk in Pune, Western India. The prevalence of DR was 14.3 percent (95\% CI 11.7 to 16.9$)$. Most diabetics $(401 / 579,69.3 \%)$ never had an eye examination for DR in the past. (Kulkarni, et al., 2019). A crosssectional study was carried out in the Department of Ophthalmology of Pravara Rural Hospital, Loni, Maharashtra from 1st October 2011 to 31st December 2011. A total of 88 diabetic patients who attended the Department of Ophthalmology of Pravara Rural Hospital, Loni, during the study period were included. The prevalence of diabetic retinopathy was 39 (44.4\%). Of the 39 patients with signs of diabetic retinopathy, $25(64.10 \%)$ were males and 14 (35.89\%) were females. Non-proliferative diabetic retinopathy was documented in $71.79 \%$, while $5.12 \%$ had proliferative diabetic retinopathy (Giri et al., 2012).

180 Diabetic patients in rural areas of South Gujarat were screened for DR between September 2014 and October 2015. The prevalence of DR was found to be $27.78 \%$. Non-Proliferative Diabetic Retinopathy (NPDR) comprised 82\%, while Proliferative Diabetic Retinopathy (PDR) comprised $18 \%$ cases. Duration of Diabetes was significantly associated with DR with prevalence in the agegroups $41-50,51-60,61-70$ and $>70$ years being $16.67 \%, 18.42 \%, 26.47 \%$ and $50 \%$, respectively (Shroff, et al., 2018).

Mehta et al., (2018) conducted a cross-sectional study from January 2013 to March 2018 on type 2 diabetic Mellitus (T2DM) subjects attending ophthalmology outpatient departments (OPDs) of G.S Randhawa Eye Hospital and Lasik Centre, Patiala, Rajindra Government Medical College and Hospital (GMCH), Patiala, J.P Eye Hospital, Zirakpur, Dhami Eye Hospital, Ludhiana and Kalia Eye Hospital, Ferozepur. These hospitals provide specialized state-of-the-art facilities for referral cases. A total of 1049 subjects were screened as T2DM according to the diagnostic criteria given by 
American Diabetes Association. The results obtained were the age range of 55-64 years, lesser number of subjects (32.77\%) had PDR in comparison to NPDR $(36.13 \%)$, however, in subjects with $>65$ years of age, this trend reversed significantly and more number of subjects $(38.98 \%)$ had PDR than NPDR (33.80\%). Similarly, subjects having $<10$ years of diabetes were more susceptible to NPDR $(60.21 \%)$ than PDR (33.90\%); however, when DOD exceeded ten years, a higher number of subjects $(66.10 \%)$ had PDR than the less severe form of NPDR (39.79\%).

Krishnaiah et al., (2007) attempted populationbased study, using a stratified random, cluster, systematic sampling strategy, in the state of Andhra Pradesh in India during 1996 and 2000. Diabetic retinopathy was present in 39 of 5586 subjects, an age-gender-area-adjusted prevalence of $0.72 \%$ (95\% confidence interval (CI): $0.49 \%-0.93 \%$ ) among subjects aged $>/=30$ years old, and $0.27 \%$ (95\% CI: $0.17 \%-0.37 \%$ ) for all ages. Most of the DR was either mild $(51.3 \%)$ or moderate $(35.9 \%)$ non-proliferative type; one subject $(2.6 \%)$ had proliferative retinopathy. A study from Telangana state was examined in a tertiary care hospital from June 2019 to Sep 2019. One hundred eight diabetic patients are included in this study, out of which the mean age of males and females were $45.61 \pm 14.21$ years and $49.68 \pm 17.16$ years. Chintala Narsaiah, et. al., (2019) found that among the 108 diabetic patients, the knowledge on diabetic retinopathy is good in only $43(39.81 \%) .65(60.19 \%)$ suffering from different severity of retinopathy. Of the 65 patients who had signs of diabetic retinopathy, there is a statistical association with age of the patients, positive family history of diabetes, poor glycaemic control, duration of diabetes, associated hypertension and smoking.

Dandona L, et al., (1999) examined 2522 subjects (85.4\% of those eligible), a representative sample of the population of Hyderabad city in southern India, underwent an interview and detailed dilated eye examination during 1996-1997 as part of the Andhra Pradesh Eye Disease Study. Diabetic retinopathy was present in 28 subjects, $1.78 \%$ (95\% CI 1.09 $2.48 \%$ ) of those $>/=30$ years old. Most of the diabetic retinopathy was of the mild $(50 \%)$ or moderate (39.3\%) non-proliferative type; one subject (3.6\%) had proliferative retinopathy. Multiple logistic regression revealed that the odds of having diabetic retinopathy were significantly higher in those $>/=50$ years than in those 30-49 years old (odds ratio 7.78, $95 \%$ CI 2.92-20.73). Three subjects had visual impairment between $6 / 12$ and $6 / 38$ in either eye due to diabetic retinopathy, $0.19 \%(95 \%$ CI $0-0.41 \%)$ of those $>/=30$ years old.

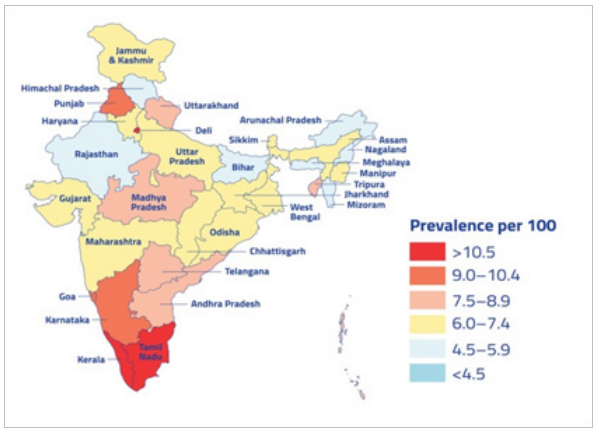

Manoj Soman et al., (2009) presented the result of 160 screening camps conducted between July 2007 and June 2008. 7321 out of 37174 people screened (19.9\%) had diabetes, including 18.9\% new diabetics. $16.2 \%$ of people with diabetes had diabetic retinopathy, including $4.3 \%$ of new diabetics. Out of 1532 gradable eyes, $86.8 \%$ had NPDR and $13.9 \%$ PDR. Vision threatening retinopathy was seen in $39.5 \%$ of eyes. FFA was advised in $34.1 \%$ of patients.

A hospital-based cross-sectional study aimed to study the prevalence of diabetic retinopathy in type 2 diabetes mellitus patients is attending the Medicine out-patient department of Government T. D. Medical College, Alappuzha, Kerala, India. The factors contributing to the development of retinopathy were also studied. 200 persons diagnosed for type 2 diabetes with the consent received, data regarding age, gender, age at onset of diabetes, duration of diabetes, history of smoking, alcohol intake, and socioeconomic status was documented. Height and weight were measured. Blood pressure was recorded with a mercury sphygmomanometer. Then the subjects were evaluated for diabetic retinopathy by fundus examination after dilating the eyes. The findings were noted and subjects were categorized as no retinopathy, nonproliferative and proliferative diabetic retinopathy using the ETDRS classification. Out of 200 subjects, 63 subjects (31.5\%) were 
affected with diabetic retinopathy (non-proliferative retinopathy $=22.5 \%$, proliferative retinopathy $=9 \%$ ). Prevalence of mild, moderate and severe nonproliferative retinopathy was $7.5 \%$ each. A significant association was found between diabetic retinopathy and duration of diabetes (Mani K., et al., 2017). A cross-sectional sample of subjects aged 50 years and older were selected using a cluster sampling technique from Palakkad district of Kerala state. Among the 5212 examined people (92\% response rate), $68(26.2 \%)$ of 260 people with a self-reported history of diabetes had diabetic retinopathy. Diabetic retinopathy among the diabetics was $26.8 \%$ (95\% CI: 19.2, 34.4, deff 1.99). Non-proliferative diabetic retinopathy $(94.1 \%)$ was the most common form of retinopathy seen. Sixty-four (94.1\%) of the 68 people diagnosed with diabetic retinopathy had NPDR of varying severity including 20 people $(29.4 \%)$ with CSMO. Twenty four $(9.2 \%)$ with diabetes required further treatment for retinopathy (Narendran et al., 2002).

Arun Kumar et al. (2017) did a cross-sectional study among 257 types 2 diabetes mellitus (DM) patients attending the Ophthalmology outpatient department at Government Medical College, Kozhikode, during a period of 6 months from January 2012 to June 2012. A total of 257 patients with diabetic retinopathy were assessed and grouped depending on the severity of retinopathy. Distribution among various stages of severity of retinopathy based on ETDRS classification was mild NPDR (Nonproliferative diabetic retinopathy) 117 , moderate NPDR 44, severe 28, very severe 32 (total NPDR 221), proliferative diabetic retinopathy (PDR) 13, high risk proliferative diabetic retinopathy (HRPDR) 20 (total PDR 33) and advanced diabetic eye disease (ADED) being 3. Another study group included 135 subjects in the same Government Medical College, Kozhikode, in 2018. There were 78 NPDR and 57 PDR cases. Those with PDR had higher age, longer duration of diabetes, lower $\mathrm{Hb}$ values, higher $\mathrm{HbA1C}$, and higher urine micro-albumin.

\section{Status of Diabetic Retinopathy in Tamil Nadu from Earlier Reviews}

A T2DM management program was initiated in the public sector in Tamil Nadu (Tony, et al.,
2016). But a growing trend in Diabetic patients in Tamil Nadu, which is understood from the following articles of different authors in a different point of time about various parts of Tamil Nadu. A cohort of 6792 NIDDM patients attending a diabetes center at Madras in South India was screened using a combination of retinal photography and clinical examination by retinal specialists. A total of 2319 patients $(34.1 \%)$ had evidence of retinopathy. Multiple logistic regression analyses showed that duration of diabetes, glycosylated hemoglobin, type of treatment (insulin treatment versus non-insulin treatment), systolic and diastolic blood pressures and serum creatinine showed a positive association with retinopathy. In contrast, body mass index (BMI) showed an inverse association (Rema et al. 1996). MV Diabetes Specialities Centre at Chennai (formerly Madras) in southern India provides treatment to approximately $15 \%$ of the diabetic population of Chennai. About $95 \%$ of patients seen at the center are drawn from southern India (Tamil Nadu, Andhrapradesh, Karnataka, and Kerala) the prevalence of retinopathy. The study group comprised 448 consecutive newly diagnosed type, 2 diabetic patients. Overall, 32 patients $(7.3 \%, 95 \%$ confidence interval (CI) 5.0 to 10.2) had diabetic retinopathy. The subjects with retinopathy had a lower BMI (mean $23.7 \mathrm{~kg} / \mathrm{m}^{2}, 95 \%$ CI 23.7 to 24.7) (Rema, et al., 2000). The Chennai Urban Rural Epidemiology Study (CURES) is a cross-sectional study that reveals the overall prevalence of DR in the population was $17.6 \%$ (95\% confidence interval [CI]: 15.8-19.5), which included 20.8\% (95\% CI: 18.7-23.1) in known diabetic subjects and 5.1\% (95\% CI: 3.1-8.0) in subjects with newly detected diabetes. The prevalence of DR was significantly higher in men than in women $(21.3 \%$ vs. $14.6 \%$; $\mathrm{P}<0.0001)$ and among subjects with proteinuria $(\mathrm{P}=0.002)$. Logistic regression analysis showed that for every 5-year increase in the duration of diabetes, the risk for DR increased 1.89-fold (95\% CI: 1.679$2.135 ; \mathrm{P}<0.0001)$. For every $2 \%$ elevation of glycated hemoglobin (HbAlc), the risk for DR increased by a factor of 1.7 (95\% CI: 1.545-1.980; $\mathrm{P}<0.0001)$ (Mohan Rema, et al., 2005). From a cross-sectional study of 1425 subjects with diabetes, 911 (63.9\%) returned for a 4-year follow-up. The incidences of 
DR, diabetic macular edema (DME), and sightthreatening diabetic retinopathy (STDR) were $9.2 \%$, $2.6 \%$, and $5.0 \%$, respectively. In subjects with DR at baseline, the incidence of DME and STDR had increased (11.5\% and 22.7\%, respectively). 1-step and 2-step progressions of DR were seen in 30.2\% and $12.6 \%$ of participants, respectively (Swati Aggarval, et al., 2005). In a cross-sectional study, a total of 26,519 participants (age $\geq 30$ years) attended 198 diabetic retinopathy screening camps conducted in three southern districts of Tamil Nadu, India, between February 2004 and April 2006. The prevalence of diabetic retinopathy was $17.6 \%$ among the self-reported rural population with diabetes. The prevalence of referable (sight-threatening) retinopathy was $5.3 \%$. Risk factors associated with the development of any DR were male gender $(\mathrm{OR}=1.37)$, longer duration of diabetes (per year, $\mathrm{OR}=1.07)$, lean body mass index $(\mathrm{OR}=1.30)$, higher systolic blood pressure (per $10 \mathrm{~mm} \mathrm{Hg}, \mathrm{OR}=1.18$ ), and insulin treatment $(\mathrm{OR}=1.34 ; \mathrm{P}<0.0001)$ (Rani, et al., 2009).

A study was conducted in the district Kanchipuram and district Thiruvallur, Tamil Nadu, India. The prevalence of any DR was higher among participants with known diabetes $(13.1 \%$ vs $2.8 \%$; participants with age between 50 and 69 years $(25.4 \%$ vs $6.2 \%$; $=0.007)$, male gender $(12.8 \%$ vs $8.1 \%$; $\mathrm{p}=0.008$ ) (Raman, et al., 2014). A cross-sectional survey among patients with T2DM attending two primary health centers for treatment and follow up in Kancheepuram, Tamil Nadu in January-March 201 stated that the prevalence of diabetic retinopathy was 29.6\% (Sharmila, et al., 2016).

A cross-sectional study was conducted among 105 Type 2 diabetic subjects in the Pakkam and Mandagapattu sub-center area of Kondur Primary Health Center in the Villupuram district of Tamil Nadu, India. The mean age of the study population was 56.69 years. About 47 (44.8\%) of the subjects were more than 60 years of age, followed by 44 subjects $(41.9 \%)$ in the age group 45-59 years. Fundus examination in at least one eye was seen in 83 people (79.0\%). Prevalence of DR in any eye and both the eye was $32.53 \%(27 / 83)$ and $31.58 \%$ (24/76), respectively. The severity of DR was moderate $(51.9 \%)$, followed by mild $(44.4 \%)$ and severe $(3.7 \%)$. DR prevalence was more among $>60$ years age group $(\mathrm{p}=0.032)$ and lesser education level $(p=0.057)$. There was no association of DR with a duration of disease, family history of diabetes, treatment regularity, presence of hypertension, visual acuity and cataract $(\mathrm{p}>0.05)$ (Balasubramaniyan, et al., 2017). The Diabetic retinopathy (DR) project, which was supported by the Queen Elizabeth Diamond Jubilee Trust, UK (the Trust), was implemented in Tirunelveli district, southern Tamil Nadu by Aravind Eye Hospital (AEH), Tirunelveli (mentoring institution). Five blocks in Tirunelveli District were selected for implementing DR Project, five $\mathrm{CHCs}$ and 99 PHCs. The project reveals the prevalence of DR among the registered Diabetes mellitus (DM population in $5 \mathrm{CHC}$ with $13 \mathrm{PHCs}$ from five blocks in the Tirunelveli district where the project was implemented. The average prevalence rate among the total DM population from all five blocks is $9 \%$. Radhapuram block has the highest prevalence rate of $10.4 \%$ comparing to other blocks. Ambasamudram block recorded the lowest prevalence rate of $7 \%$. The prevalence of DR in Manor block is $8.5 \%$, in Pappakudi block is 9.3\%, and in Kalakadu is 9.8\% (Report Amaltas 2019).

\section{Conclusion}

Agarwal, S., etal. (2006) found that the prevalence rate of Diabetic Retinopathy was 6.35 percent in Tamil Nadu. According to Namperumalsamy, et al., (2009), the prevalence rate of Diabetic Retinopathy in (a district of Tamil Nadu) Theni, Tamil Nadu was $12.2 \%$. Raman, R., et al., (2009) study depicted that the prevalence rate was 18 percent in Chennai, Tamil Nadu, 18\%. The study based on Sankara Nethralaya Chennai by Raman R et al. (2012) stated that the prevalence rate of Diabetic retinopathy in Tamil Nadu was 4.8\%. Balasubramaniyan Natarajan (2017) et al., found that the prevalence rate of diabetic retinopathy was 32.5 percent in Villupuram District of Tamil Nadu. Based on the report of Amaltas 2019, the prevalence rate per 100 of Diabetic Retinopathy is greater than 10.5 in Tamil Nadu and highest among the states in India. Hence, it is an urgent need to take care of Diabetic patients specifically older age people, to mitigate Diabetic Retinopathy. The issue of Diabetic Retinopathy impacts not only 
the individual but his/her social capital and will be obstacles for involving in productive/economic activities.

\section{References}

Agarwal, Swati, et al. "Sankara Nethralaya Diabetic Retinopathy Epidemiology and Molecular Genetic Study (SN - DREAMS 1): Study Design and Research Methodology.' Ophthalmic Epidemiology, vol. 12, no. 2, 2005, pp. 143-153.

Ayoor, Arun Kumar, et al. "Association of Severity of Diabetic Retinopathy with Comorbidities in Type 2 Diabetes Mellitus." Indian Journal of Clinical and Experimental Ophthalmology, vol. 3, no. 4, 2017, pp. 436-439.

Dandona, Lalit, et al. "Population Based Assessment of Diabetic Retinopathy in an Urban Population in Southern India." British Journal of Ophthalmology, vol. 83, no. 8, 1999, pp. 937-940.

Gadkari, Salil S., et al. "Prevalence of Diabetic Retinopathy in India: The All India Ophthalmological Society Diabetic Retinopathy Eye Screening Study 2014." Indian Journal of Ophthalmology, vol. 64, no. 1, 2016, pp. 38-44.

Giri, Purushottam A., et al. "Prevalence of Diabetic Retinopathy and Associated Risk Factors among Diabetic Patients Attending Pravara Rural Hospital, Loni, Maharashtra." Journal of Academy of Medical Sciences, vol. 2, no. 2, 2012, pp. 64-67.

India: Health of the Nation's States. Department of Health Research, Government of India, 2017.

International Diabetes Federation, Diabetes Atlas. 2019.

Jotheeswaran, A.T., et al. "Estimating the Proportion of Persons with Diabetes Developing Diabetic Retinopathy in India: A Systematic Review and Meta-Analysis." Indian Journal of Endocrinology and Metabolism, vol. 20, 2016, pp. 51-58.

Krishnaiah, Sannapaneni, et al. "Risk Factors for Diabetic Retinopathy: Findings from the Andhra Pradesh Eye Disease Study." Clinical Ophthalmology, vol. 1, 2007, pp. 475-482.
Kulkarni, Sucheta, et al. "Estimating the Magnitude of Diabetes Mellitus and Diabetic Retinopathy in an Older Age Urban Population in Pune, Western India." BMJ Open Ophthalmology, vol. 4, no. 1, 2019.

Mani, Koushiki, and Rose Davy C. "Prevalence of Diabetic Retinopathy in Type 2 Diabetes Mellitus Patients Attending Medicine OutPatient Department of a Tertiary Care Hospital in Alappuzha, Kerala, India." International Journal of Research in Medical Sciences, vol. 5, no. 4, 2017, pp. 1532-1536.

Mehta, Kanchan, et al. "Prevalence and Predictors of Diabetic Retinopathy in the Population of Punjab: North Indian Diabetic Retinopathy Epidemiology and Molecular Genetic Study (Ni-Dreams)." International Journal of Health Sciences and Research, vol. 8, no. 9, 2018, pp. 1-9.

Mohammed, Thasni, et al. "Predictors of Severity of Retinopathy among Subjects with Early Onset Type 2 Diabetes Mellitus." Indian Journal of Clinical and Experimental Ophthalmology, vol. 4, no. 4, 2018, pp. 478-482.

Nadarajan, Balasubramanian, et al. "Prevalence of Diabetic Retinopathy and its Associated Factors in a Rural Area of Villupuram District of Tamil Nadu, India." Journal of Clinical and Diagnostic Research, vol. 11, no. 7, 2017.

Narendran, V., et al. "Diabetic Retinopathy among Self-Reported Diabetics in Southern India: A Population Based Assessment." British Journal of Ophthalmology, vol. 86, 2002, pp. 1014-1018.

Narsaiah, Chintala, et al. "Study on Awareness and Assessment of Diabetic Retinopathy in Diabetic Patients Attending Ophthalmology Clinic at a Tertiary Care Hospital, Telangana State." International Journal of Contemporary Medical Research, vol. 6, no. 11, 2019.

NCD Risk Factor Collaboration. "Worldwide Trends in Diabetes since 1980: A Pooled Analysis of 751 Population-Based Studies with $4 * 4$ Million Participants." Lancet, 2016.

Piloting the Treatment of Retinopathy in India: Diabetic Retinopathy and Retinopathy of Prematurity. Amaltas, 2019. 
Qureshi, Tariq, et al. "Prevalence of Diabetic Retinopathy in Kashmir, India - A Hospital based study Global." Journal of Medicine and Public Health, 2013.

Rema, Mohan, et al. "Prevalence of Diabetic Retinopathy in Urban India: The Chennai Urban Rural Epidemiology Study (CURES) Eye Study, I." Investigative Ophthalmology \& Visual Science, vol. 46, no. 7, 2005, pp. 2328-2333.

Rema, Mohan, et al. "Prevalence of Retinopathy at Diagnosis among Type 2 Diabetic Patients attending a Diabetic Centre in South India." British Journal of Ophthalmology, vol. 84, 2000, pp. 1058-1060.

Saeedi, Pouya, et al. "Global and Regional Diabetes Prevalence Estimates for 2019 and Projections for 2030 and 2045: Results from the International Diabetes Federation Diabetes Atlas, 9th edition." Diabetes Research and Clinical Practice, vol. 157, 2019.
Shroff, Pavan Humesh, et al. "Prevalence of Diabetic Retinopathy (DR) in Rural Areas of Western India." National Journal of Community Medicine, vol. 9, no. 9, 2018, pp. 647-650.

Soman, Manoj, et al. "Population Based Assessment of Diabetes and Diabetic Retinopathy in South Kerala-Project Trinetra: An Interim Report." Kerala Journal of Ophthalmology, vol. XXI, no. 1, 2009, pp. 36-41.

Wild, Sarah, et al. "Global Prevalence of Diabetes: Estimates for the Year 2000 and Projections for 2030." Diabetes Care, vol. 27, 2004, pp. 1047-1053.

Yang, Qian-Hui, et al. "Prevalence of Diabetic Retinopathy, Proliferative Diabetic Retinopathy and Nonproliferative Diabetic Retinopathy in Asian T2DM Patients: A Systematic Review and Meta-analysis." International Journal of Ophthalmology, vol. 12, no. 2, 2019, pp. 302-311.

\author{
Author Details \\ Dr. M. Chitra, Assistant Professor, Department of Econometrics, Madurai Kamaraj University, Madurai, \\ Tamil Nadu, India, Email ID: chitra.eco@mkuniversity.org
}

Dr. Aruthdha Shree Neevanandam, Nantong University, Nantong, Jiangsu, China 